\title{
Realization and detection of Weyl semimetals and the chiral anomaly in cold atomic systems
}

\author{
Wen-Yu He, ${ }^{1}$ Shizhong Zhang, ${ }^{2}$ and K. T. Law ${ }^{1,3, *}$ \\ ${ }^{1}$ Department of Physics, Hong Kong University of Science and Technology, Clear Water Bay, Hong Kong, China \\ ${ }^{2}$ Department of Physics and Center of Theoretical and Computational Physics, University of Hong Kong, Hong Kong, China \\ ${ }^{3}$ Center for $2 D$ and $1 D$ Quantum Materials, Hong Kong University of Science and Technology, Clear Water Bay, Hong Kong, China
}

(Received 20 February 2015; revised manuscript received 22 June 2016; published 13 July 2016)

\begin{abstract}
In this work, we describe a method to realize a three-dimensional Weyl semimetal by coupling multilayers of a honeycomb optical lattice in the presence of a pair of Raman lasers. The Raman lasers render each isolated honeycomb layer a Chern insulator. With finite interlayer coupling, the bulk gap of the system closes at certain out-of-plane momenta due to Raman assisted tunneling and results in the Weyl semimetal phase. Using experimentally relevant parameters, we show that both one pair and two pairs of Weyl points can be realized by tuning the interlayer coupling strength. We suggest that Landau-Zener tunneling can be used to detect Weyl points and show that the transition probability increases dramatically when the Weyl point emerges. The realization of chiral anomaly by using a magnetic-field gradient is also discussed.
\end{abstract}

DOI: 10.1103/PhysRevA.94.013606

\section{INTRODUCTION}

The search for topological phases that are fully gapped in the bulk and possess topologically protected gapless surface states has been an important subject in physics. Several new topological phases, such as two-dimensional (2D) and threedimensional (3D) topological insulators [1,2] and quantum anomalous Hall insulators [3], have been realized experimentally in condensed-matter systems. The gapless surface states in these systems are protected by certain symmetries and the bulk gap [1,2]. Nevertheless, the surface states can be removed once the bulk gap in the system is closed.

Interestingly, it is well known that Weyl semimetals [4-8], which contain nodal points, the Weyl points, in the energy spectrum also support topologically protected gapless Fermi arcs on the surface. A pair of Weyl points in a Weyl semimetal can be regarded as a pair of topological defects in 3D momentum space with opposite chirality and possesses a linear energy spectrum near the Weyl points. The Fermi arcs, which connect the Weyl points in the surface Brillouin zone, cannot be removed unless the Weyl points are merged together by strong perturbations [4,5]. Due to the presence of the Weyl points in the bulk and the gapless Fermi arcs on the surface, Weyl semimetals are expected to exhibit interesting novel phenomena which are absent in fully gapped topological phases such as the chiral anomaly $[9,10]$ and unconventional quantum oscillations [11]. Recently the experimental realization of the Weyl semimetal phase has been widely studied in real materials $[12,13]$.

On the other hand, great advancement has been made in realizing topological phases with cold atoms in optical lattices. Using Raman laser coupling [14-20] and an optical shaking lattice [21-23], Chern insulators have been successfully realized experimentally [24,25] using both schemes. Methods for realizing 3D topological insulators in optical lattices have also been proposed [26,27]. In this work, we outline a practical scheme to realize and detect the Weyl semimetal phase in optical lattices.

\footnotetext{
*phlaw@ust.hk
}

We base our proposal on the already realized honeycomb optical lattice $[28,29]$ and show that by introducing nontrivial hopping between the neighboring honeycomb lattice layers it is possible to generate either one pair or two pairs of Weyl points in the system. What is important in our condition is that the momentum transfer $\Delta \mathbf{p}$ from Raman beams possesses nonzero components both along and perpendicular to the honeycomb lattice planes. Within each individual lattice layer, in-plane momentum transfer generates nontrivial Peierls phases and can drive each layer into a 2D Chern insulator phase. Importantly, when individual layers are coupled with nontrivial phases generated by the Raman beams, the Chern insulators can become trivial for certain out-of-plane momentum $k_{z}$. Since the bulk gap has to be closed for topological phase transitions from the topologically nontrivial Chern insulators to trivial insulators as a function of out-of-plane momenta $k_{z}$, there are Weyl points in the momentum space separating the trivial and nontrivial regimes. The number of Weyl points, corresponding to the number of the band-gap closing points as a function of $k_{z}$, is tunable by changing, for example, the Raman detuning and the interlayer coupling. We suggest that Landau-Zener transitions can be used to detect the emergence of the Weyl points. We also discuss how Landau levels and the chiral anomaly can be realized by introducing a magnetic field gradient in the $z$ direction.

\section{THE SCHEME}

We first consider a multilayer honeycomb optical lattice generated by laser beams [28,29]:

$$
\begin{aligned}
V= & -V_{\bar{X}} \cos ^{2}(q \hat{x}+\theta / 2)-V_{X} \cos ^{2}(q \hat{x})-V_{Y} \cos ^{2}(q \hat{y}) \\
& -2 \alpha \sqrt{V_{X} V_{Y}} \cos (q \hat{x}) \cos (q \hat{y})-V_{\bar{Z}} \cos ^{2}\left(q_{z} \hat{z}\right)
\end{aligned}
$$

where $V_{X}, V_{\bar{X}}$, and $V_{Y}$ are intensities of lasers $X, \bar{X}$, and $Y$, pointing along $\hat{x},-\hat{x}$, and $\hat{y}$ directions, respectively. $q$ is the wave vector of the lasers. When $V_{\bar{X}} \gg V_{Y} \geq V_{Z}$, a honeycomb lattice is created with two lattice sites $A$ and $B$ in a unit cell, as shown in Fig. 1(a). The energy offset $\Delta$ between $A$ and $B$ sublattices can be tuned through the phase difference $\theta$ between $X$ and $\bar{X}$ lasers. The standing wave $\bar{Z}$, with wave 


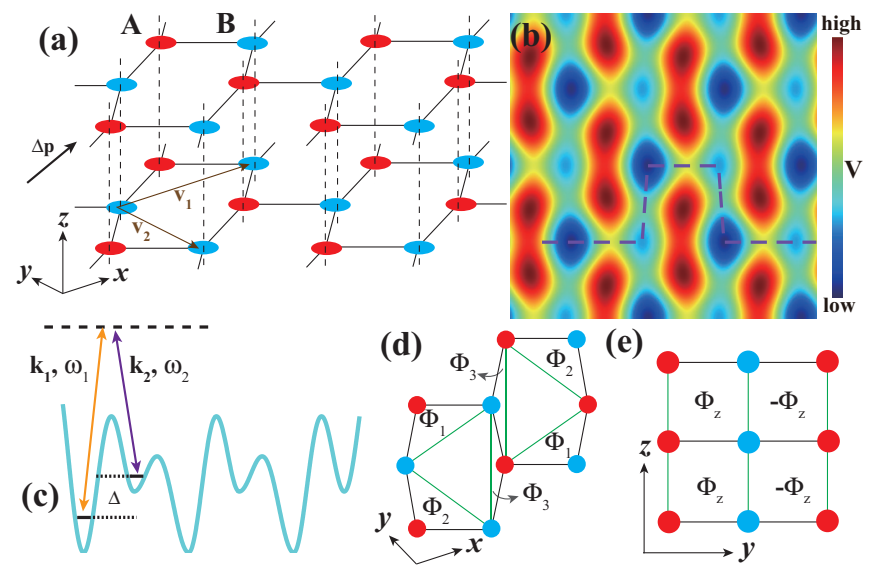

FIG. 1. (a) $A A$ stack of the multilayer honeycomb optical lattice. (b) The intralayer optical potential. The bigger dark blue and the smaller light blue pockets represent the $A$ and $B$ sites, respectively. (c) The Raman laser assisted resonant tunneling between nearest neighbors. The recoil momentum carried by photons generates Peierls phases in the hopping amplitudes. (d) The synthetic magnetic flux $\Phi_{i}$ of each triangle resulting from the Peierls phases in the $x y$ plane. (e) The synthetic magnetic flux of each rectangle in the $y z$ plane.

vector $q_{z}$, stacks the honeycomb optical lattice as seen in Fig. 1(a), and controls the interlayer coupling strength. The energy offset $\Delta$ is tuned to be large enough such that the direct tunneling between neighboring $A$ and $B$ sites is greatly suppressed in the absence of Raman beams.

A pair of Raman beams with frequency detuning $\delta \omega \equiv$ $\omega_{1}-\omega_{2} \approx \Delta / \hbar$ is added to restore the resonant tunneling between neighboring intralayer sites, as illustrated in Fig. 1(c). The momentum transfer $\Delta \mathbf{p}=\mathbf{k}_{1}-\mathbf{k}_{2}$ associated with the resonant tunneling introduces Peierls phases $\exp \left[i \Delta \mathbf{p} \cdot\left(\mathbf{r}+\mathbf{r}^{\prime}\right) / 2\right]$ between intralayer neighboring sites $\mathbf{r}$ and $\mathbf{r}^{\prime}$. This introduces synthetic magnetic flux into the optical lattices [19,30] as depicted in Fig. 1(d). Using Floquet analysis and a unitary transformation which eliminates the spatial dependence of the Peierls phases (for details, see Appendix A), the effective Hamiltonian can be written as

$$
\begin{aligned}
H= & \sum_{\langle i, j\rangle, l}\left(t_{i j}^{0} \tilde{b}_{i, l}^{\dagger} \tilde{a}_{j, l}+t_{i j}^{a} e^{i \phi_{i j}} \tilde{a}_{i, l}^{\dagger} \tilde{a}_{j, l}+t_{i j}^{b} e^{-i \phi_{i j}} \tilde{b}_{i, l}^{\dagger} \tilde{b}_{j, l}\right) \\
& +\epsilon \sum_{i, l}\left(\tilde{a}_{i, l}^{\dagger} \tilde{a}_{i, l}-\tilde{b}_{i, l}^{\dagger} \tilde{b}_{i, l}\right)+\sum_{i, l}\left(t_{\perp}^{a} e^{i \phi_{\perp}} \tilde{a}_{i, l}^{\dagger} \tilde{a}_{i, l+1}\right. \\
& \left.+t_{\perp}^{b} e^{-i \phi_{\perp}} \tilde{b}_{i, l}^{\dagger} \tilde{b}_{i, l+1}\right)+ \text { H.c. }
\end{aligned}
$$

Here, $i, j$ labels the position of lattice sites within a layer and $l$ is the layer index. $\tilde{a}_{i, l}\left(\tilde{b}_{i, l}\right)$ annihilates an atom at sublattice $A(B)$ of site $i$ and layer $l . t_{i j}^{0}$, which satisfies $t_{i, i-1}^{0}=-t_{i, i+1}^{0}$ due to the Raman fields [31], denotes the intralayer nearest-neighbor hopping amplitude and $t_{i j}^{a}\left(t_{i j}^{b}\right)$ is the next-nearest-neighbor hopping amplitude between $A(B)$ sublattice sites. $t_{\perp}^{a}$ and $t_{\perp}^{b}$ are the interlayer hopping amplitudes and $\epsilon=\frac{1}{2}(\hbar \delta \omega-\Delta)$, originating from the Raman detuning, can be regarded as the on-site energy difference between the sublattices. The Peierls phases $\phi_{i j}$ and $\phi_{\perp}$ are associated with intralayer and interlayer hopping, generated by the in-plane and the out-of-plane components of $\Delta \mathbf{p}$, respectively. Explicitly, one finds

$$
\phi_{i j}=\frac{1}{2} \Delta \mathbf{p} \cdot\left(\mathbf{r}_{i, l}-\mathbf{r}_{j, l}\right), \phi_{\perp}=\frac{1}{2} \Delta \mathbf{p} \cdot\left(\mathbf{r}_{i, l}-\mathbf{r}_{i, l+1}\right) .
$$

In particular, $\phi_{i j}$ is responsible for making each isolated layer of honeycomb lattices a Chern insulator, and $\phi_{\perp}$, which originates from the phase difference between adjacent layers due to nonzero vertical momentum transferred in the Raman process, induces unconventional interlayer coupling, which is the key for generating the Weyl semimetal phase. The accumulation of Peierls phases along closed paths gives rise to synthetic magnetic flux patterns as shown in Figs. 1(d) and 1(e).

\section{THE WEYL POINTS}

To illustrate the emergence of Weyl points, we first consider an ideal version of the experimental honeycomb lattice (brickwall lattice). For simplicity, we set $\left|t_{i j}^{0}\right|=t$ and $t_{i j}^{a}=t_{i j}^{b}$ (in the following, next-nearest-neighbor hopping $t_{1}$ is along $\mathbf{v}_{1}$ or $\mathbf{v}_{2}$ while $t_{2}$ is along $\mathbf{v}_{1}-\mathbf{v}_{2}$ ). We use the coordinate system established as shown in Fig. 1(a) for convenience and the momentum transfer is set to be $\Delta \mathbf{p}=\left(p_{x}, p_{y}, p_{z}\right)$. If we further label the $A(B)$ sublattices as corresponding to a pseudospin $\sigma$, then the Hamiltonian takes the following form in momentum space:

$$
H(\mathbf{k})=d_{0}(\mathbf{k}) I+\mathbf{d}(\mathbf{k}) \cdot \boldsymbol{\sigma}
$$

with

$$
\begin{aligned}
d_{0}(\mathbf{k})= & 2 t_{1} \cos k_{x} a \cos \frac{1}{2} p_{x} a+2 t_{1} \cos k_{y} a \cos \frac{1}{2} p_{y} a \\
& +2 t_{2} \cos \left(k_{x}+k_{y}\right) a \cos \frac{1}{2}\left(p_{x}+p_{y}\right) a \\
& +2 t_{\perp} \cos k_{z} d \cos \phi_{\perp}, \\
d_{x}(\mathbf{k})= & t \cos \frac{1}{2}\left(k_{x}-k_{y}\right) a, \\
d_{y}(\mathbf{k})= & -2 t \sin \frac{1}{2}\left(k_{x}+k_{y}\right) a+t \sin \frac{1}{2}\left(k_{x}-k_{y}\right) a, \\
d_{z}(\mathbf{k})= & 2 t_{1} \sin k_{x} a \sin \frac{1}{2} p_{x} a+2 t_{1} \sin k_{y} a \sin \frac{1}{2} p_{y} a \\
& +2 t_{2} \sin \left(k_{x}+k_{y}\right) a \sin \frac{1}{2}\left(p_{x}+p_{y}\right) a \\
& -2 t_{\perp} \sin k_{z} d \sin \phi_{\perp}+\epsilon,
\end{aligned}
$$

where $a$ and $d$ are lattice constants in the $x y$ plane and along the $\hat{z}$ axis. A $k_{z}$-dependent Zeeman term $m=\epsilon-$ $2 t_{\perp} \sin k_{z} d \sin \phi_{\perp}$ appears in the effective Zeeman fields along the $\hat{z}$ direction. For fixed $m$, the Chern number

$$
C=\frac{1}{4 \pi} \int d k_{x} d k_{y} \hat{d}(\mathbf{k}) \cdot\left[\partial_{x} \hat{d}(\mathbf{k}) \times \partial_{x} \hat{d}(\mathbf{k})\right]
$$

can be defined as the topological invariant of the system with fixed $k_{z}$ and $\hat{d}(\mathbf{k}) \equiv \mathbf{d}(\mathbf{k}) /|\mathbf{d}(\mathbf{k})|$.

The phase diagram of an effective $2 \mathrm{D}$ system with $\Delta \mathbf{p}=$ $\left(0, p_{y}, 0\right)$ as a function of fixed $m$ and $p_{y}$ is summarized in Fig. 2(a), where the colored region has a nonzero Chern number. When the interlayer coupling $t_{\perp}$ is zero, $m=\epsilon$ with $p_{y} a \in(0,2 \pi) \cup(2 \pi, 4 \pi)$ makes $\left|d_{z}\right|>0$ for all $k_{z}$. As a result, the system is fully gapped in the bulk.

As the interlayer coupling is increased to a finite value, $m$ as well as the bulk gap change as a function of $k_{z}$. From Eq. (5), it is clear that the Weyl points appear when $\left(k_{x} a, k_{y} a\right)=$ $\left(-\frac{2}{3} \pi,-\frac{5}{3} \pi\right)$ or $\left(k_{x} a, k_{y} a\right)=\left(\frac{2}{3} \pi,-\frac{1}{3} \pi\right)$ and at a value of $k_{z}$ 

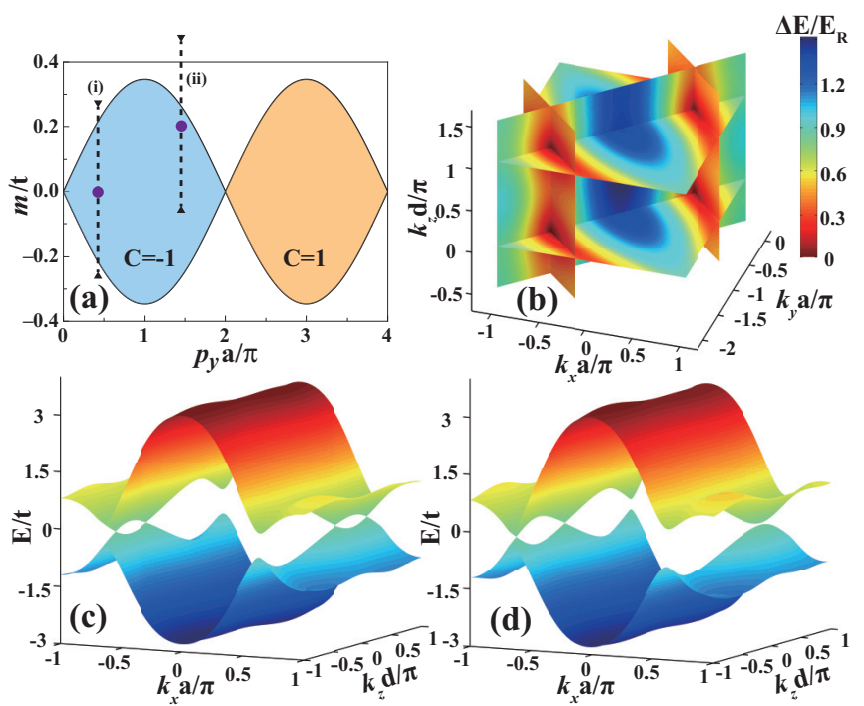

FIG. 2. (a) The Chern number $C$ of an isolated layer with $t_{2}=3 t_{1}=0.3 t$ as a function of the Zeeman term $m$ and recoil momentum $\Delta \mathbf{p}=\left(0, p_{y}, 0\right)$. The purple dots denote $m\left(k_{z}=0\right)=\epsilon$, while the dashed lines correspond to the range of $m$ for general $k_{z}$ when interlayer coupling is turned on. (b) The energy difference between the upper and the lower bands, using parameters from a realistic optical lattice, is shown. It is evident that two pairs of Weyl points locate in the plane of $k_{y}=k_{x}-\pi$. (c) Energy spectrum in the $k_{y}=k_{x}-\pi$ plane with $\epsilon=0$ where two pairs of Weyl points emerge. (d) One pair of Weyl points emerges with $\epsilon=0.2 t$. In (c) and (d) we take $t_{1}=0.1 t, t_{2}=0.3 t, t_{\perp}=0.26 t$, and $\Delta \mathbf{p}=(0, \pi / a, \pi / d)$ for calculation.

where $d_{z}\left(k_{z}\right)=0$ such that $|\mathbf{d}|=0$. For systems with specific $t_{\perp}$ and $\Delta \mathbf{p}$, the ranges of $m\left(k_{z}\right)$ as a function of $k_{z}$, where $k_{z}$ ranges from $-\pi / d$ to $\pi / d$, are schematically indicated as vertical dashed lines (i) for $\epsilon=0$ and (ii) for $\epsilon=0.2 t$ in Fig. 2(a). The number of Weyl points is determined by the number of crossings of the dashed lines and the topological phase boundary in Fig. 2(a). The crossing is controllable by tuning the interlayer coupling, the detuning and the momentum transfer $\Delta \mathbf{p}$ in the Raman process, so the Weyl semimetal phase with either one pair or two pairs of Weyl points becomes available. The energy spectra for systems which contain one pair or two pairs of Weyl points are depicted in Figs. 2(c) and 2(d), respectively. The ranges of the Zeeman terms $m$ as a function of $k_{z}$ in Figs. 2(c) and 2(d) correspond to the vertical lines (i) and (ii) in Fig. 2(a), respectively.

In the following, we consider a realistic optical potential with $\theta=\frac{2}{3} \pi$ and $V_{[\bar{X}, X, Y]}=[7,0.5,2] E_{R}$, where $E_{R} \equiv q^{2} / 2 m$ is the recoil energy [28,29]. The hopping parameters of the optical lattices are calculated from the maximally localized Wannier function method (see Fig. 8). In realistic band structures with the Raman laser strength $V_{0}=E_{R}$, two pairs of Weyl points emerge successively when the amplitude of $V_{Z}$ is increased. Using $t_{\perp}=0.09 E_{R}, \Delta \mathbf{p}=(0,2 \pi / a, \pi / d)$, and $\epsilon=$ 0 , we plot the energy gap between the lower and the upper bands in Fig. 2(b). Here the energy gap is plotted for the planes $k_{z}=0, k_{z}=\pi, k_{y}=k_{x}-\pi$, and $k_{y}=-k_{x}+k_{x i}+k_{y i}$ with $i=1,2$. The four crossing points $\left(k_{x i}, k_{y i}, 0\right)$ and $\left(k_{x i}, k_{y i}, \pi\right)$ between the orthogonal planes are the Weyl points. It is evident that the four band touching points represent the Weyl points. It is important to note that, using the realistic parameters, there is a wide range of experimentally accessible parameter space of $E_{R}$ and $V_{z}$ in which the Weyl semimetal phase can be realized.

\section{DETECTION OF THE WEYL SEMIMETAL PHASE}

Our proposed scheme for realizing the Weyl semimetal consists of two steps: (1) create isolated 2D Chern insulators and (2) couple the isolated layers with nontrivial hoppings. To confirm the emergence of the Weyl phase, we shall first detect the Chern insulator and then ascertain the closing of the bulk gap when interlayer tunneling is increased. As we show below, both can be carried out with Bloch oscillations.

It is important to note that the nonzero Chern number comes from the integral of Berry curvature over the first Brillouin zone. Thus one way to reveal the topological order is to measure the Berry curvature, as was proposed theoretically in $[32,33]$ and performed experimentally $[28,29]$. In the Bloch oscillation induced by a constant force, the atom cloud, in addition to the group velocity parallel to the force, would obtain an anomalous velocity that is transverse to the force due to nonzero Berry curvature [34]. After one full Bloch cycle, this Berry curvature generated anomalous velocity would slightly drive the atom cloud transversely. This transverse drift, which is measurable experimentally, can manifest the topological order of Chern insulators.

After the confirmation of the 2D Chern insulator phase for the isolated layers, the interlayer coupling can be gradually enhanced through modulating the intensity of laser $\bar{Z}$ to close the bulk band gap [29]. Such band-gap closing can be monitored by the fraction of atoms in the excited band after a cycle of Bloch oscillations, as was done for an isolated 2D honeycomb lattice $[28,35]$. Below, we extend this technique to the Weyl semimetal case and calculate the Landau-Zener probability.

More specifically, we use the model described by $H(\mathbf{k})$ in Eq. (4) and consider an external linear potential applied along the (110) direction where there are two pairs of Weyl points [see Fig. 3(a)]. The total probability for interband transition will come from two independent Landau-Zener events as schematically depicted in Fig. 3(b). Since the transition probability in each Landau-Zener event increases exponentially as the gap decreases, the emergence of Weyl points is accompanied by a dramatic increase in the transferred fraction when the bulk band gap closes. For noninteracting fermionic atoms, as was performed in a recent experiment [28], the Landau-Zener probability must be averaged over the initial distribution of atoms [35].

Let us denote the energy of the lowest band as $E(\mathbf{k})$ and the energy difference between the lowest and the first excited bands close to the band touching point as $\delta(\mathbf{k})$. Then, within local-density approximation, the Landau-Zener transition probability can be written as

$$
P=\frac{\int_{E(\mathbf{k}, \mathbf{r}) \leqslant E_{F}} p(\mathbf{k}) d^{3} \mathbf{k} d^{3} \mathbf{r}}{\int_{E(\mathbf{k}, \mathbf{r}) \leqslant E_{F}} d^{3} \mathbf{k} d^{3} \mathbf{r}},
$$

where $E_{F}$ is the Fermi energy and $E(\mathbf{k}, \mathbf{r})=E(\mathbf{k})+V(\mathbf{r})$ with $V(\mathbf{r})$ the external harmonic confining potential. $p(\mathbf{k})$ is the 


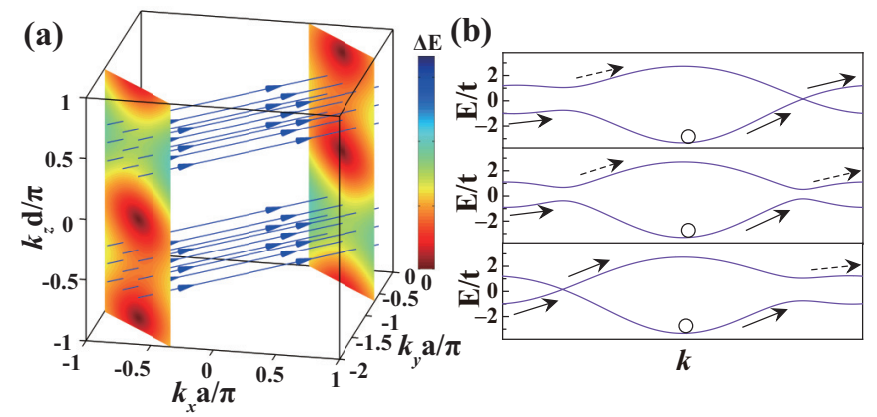

FIG. 3. (a) The two planes which contain the Weyl points are depicted. The color represents the energy difference between the upper band and lower band and indicates the position of the Weyl points. Driven in the (110) direction, atoms at the bottom of the lower band will move along the arrows in the $\mathbf{k}$ space and penetrate the two planes. Atoms in a unit Bloch oscillation encounter two local band-gap minima and undergo two Landau-Zener tunneling events. (b) Three typical cases of Landau-Zener transition. The path in the upper and bottom panel contains Weyl points with different locations, while that in the middle panel is fully gapped. The dashed arrow denotes that the Landau-Zener transition probability is small due to the existence of the energy gap.

Landau-Zener probability for the $\mathbf{k}$ state:

$$
p(\mathbf{k})=\sum_{i \neq j} \exp \left[-\frac{\pi \delta_{i}^{2}(\mathbf{k})}{4 v_{i} F}\right]\left(1-\exp \left[-\frac{\pi \delta_{j}^{2}(\mathbf{k})}{4 v_{j} F}\right]\right),
$$

where $i(j)$ labels the corresponding Landau-Zener events along the (110) direction and $v_{i(j)}$ is the velocity close to Weyl points. $F$ is the strength of the linear potential.

The transition probability as a function of $t_{\perp}$, for systems with different $E_{F}$, is shown in Fig. 4(a). The momentum transfer due to the Raman field is $p_{y} a=\pi$ and $p_{z} d=\pi$ such that the system can be a Weyl semimetal when $t_{\perp}$ is larger than some critical values. Initially, atoms with energy lower than $E_{F}$ are occupied near the $\vec{k}=(0,0, \pm \pi / 2 d)$. For small $t_{\perp}$, the system is fully gapped and the Landau-Zener transition probability is exponentially small. At certain critical $t_{\perp}$, Weyl points emerge on the line along the (110) direction. Due to the gap closing, the interband transition probability increases dramatically as shown in Fig. 4(a). However, by further increasing $t_{\perp}$, the Weyl points move away from the $\left|k_{z} d\right|=\pi / 2$ plane. As a result, the atoms driven in the (110) direction will not be able to access the Weyl points and the transition amplitude decreases. Therefore, there is a peak in the transition probability as a function of $t_{\perp}$ and the peak location indicates the critical value of $t_{\perp}$ at which the Weyl points emerge. In other words, the transition peak separates the fully gapped Chern insulator phase from the nodal Weyl semimetal phase.

The density plot of the transition probabilities as a function of $t_{\perp}$ and $p_{y}$ for Fermi energy $E_{F}=0.05 t$ is shown in Fig. 4(b). The Landau-Zener transition peak locates around $t_{\perp}=\frac{\sqrt{3}}{2}\left|\sin \frac{1}{2} p_{y} a\left(t_{1}-t_{2}\right)\right|$, which is denoted as the dashed line in Fig. 4(b). As in Fig. 4(a), the peak separates the Chern insulator phase from the Weyl semimetal phase. Similar results can be obtained when realistic parameters are used.

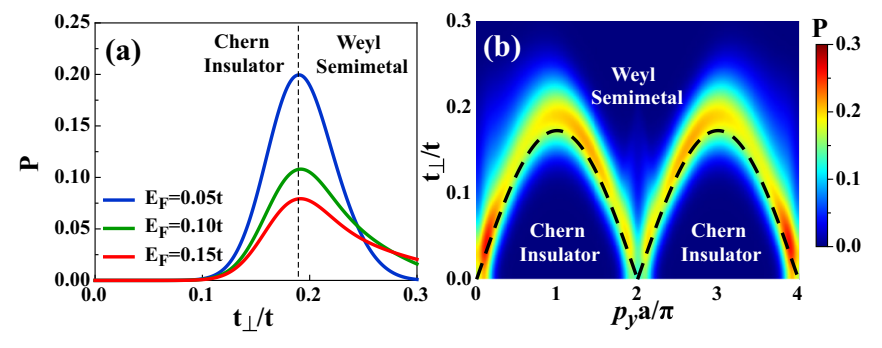

FIG. 4. (a) Landau-Zener transition probabilities as a function of $t_{\perp}$ for different Fermi energies. A transition peak signals the emergence of Weyl points at the critical value of $t_{\perp}$. The peak separates the fully gapped Chern insulator phase from the Weyl semimetal phase. (b) Landau-Zener transition probability as a function of both $t_{\perp}$ and $p$ with Fermi energy $E_{F}=0.05 t$. In calculations, we take $F=0.01, t_{1}=0.1 t, t_{2}=0.3 t, \epsilon=0$, and $\Delta \mathbf{p}=(0, \pi / a, \pi / d)$.

\section{LANDAU LEVELS AND CHIRAL ANOMALY}

It is important to note that the Weyl semimetal phase discussed above can be used to realize the chiral anomaly. Considering a low-energy Weyl Hamiltonian without any velocity anisotropy for simplicity, in the presence of an external magnetic field $\mathbf{B}=\left(B_{x}, 0,0\right)$, we can choose the Landau gauge $\mathbf{A}=(0,0, e B y)$ to obtain $H_{\mathrm{W}}=$ $\chi v_{F}\left[\delta k_{x} \sigma_{x}-i \partial_{y} \sigma_{y}+\left(\delta k_{z}+e B y\right) \sigma_{z}\right]$, where $\chi= \pm 1$ represents the chirality of the Weyl point. Along the direction parallel to the magnetic field, the infinite Landau bands form gapped spectrum $E_{n}=\hbar v_{F} \operatorname{sgn}(n) \sqrt{2|n| e B / \hbar+\delta k_{x}^{2}}$, with $n= \pm 1, \pm 2, \ldots$ and also gapless chiral modes $E_{0}=\chi v_{F} \delta k_{x}$. The gapless chiral modes induced by the zeroth Landau levels have opposite group velocity for the states at Weyl points of opposite chirality. When a constant force (gradient scalar field) is applied parallel to the magnetic field, states near one Weyl point can be driven to the other Weyl point of opposite chirality through the gapless chiral modes. In this case the particle number is not conserved in a single Weyl point but the total particle number over the two Weyl points remains a constant. In the background of the magnetic field and gradient scalar field, this violation of particle number in a single Weyl point of specific chirality is referred to as a chiral anomaly [36]. In the following, we will show that a uniform synthetic magnetic field can be introduced in our Weyl semimetal phase to form the chiral Landau bands and further simulate the chiral anomaly.

In our $A A$ stacked honeycomb optical lattice, when a linear magnetic-field gradient is induced along the $z$ direction and together with the laser assisted tunneling, an extra phase $\Phi=\Delta \mathbf{p} \cdot \mathbf{r}_{i, j, l}=i \phi_{x}+j \phi_{y}+l \phi_{z}$ is added to the $z$ direction hopping $t_{\perp}^{a(b)}$ [19]. In our choice of Raman lasers, $\left(\phi_{x}, \phi_{y}, \phi_{z}\right)=$ $\left(0, \frac{\pi}{2}, \pi\right)$, the extra phase $\Phi$ results in a uniform synthetic magnetic field along the $x$ direction, as it is schematically shown in Fig. 5(a).

In the presence of this uniform magnetic field along the $x$ direction, the energy spectrum formed by Landau levels is generated. The zeroth Landau levels result in gapless chiral modes along the $k_{x}$ direction at the Weyl points. The chiralities of the gapless chiral modes are determined by the chiralities of the Weyl points [11]. The energy levels in the presence of the synthetic magnetic flux as a function of $k_{x}$ are depicted in 


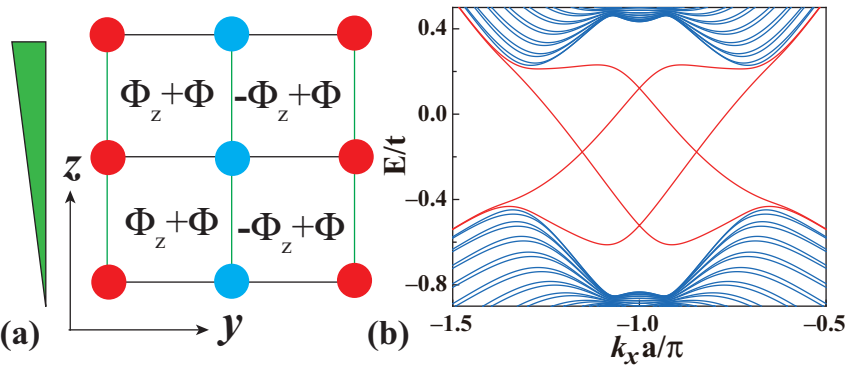

FIG. 5. (a) Schematic illustration of uniform magnetic flux $\Phi$ generated by a magnetic-field gradient in the $z$ direction and the Raman laser assisted tunnelings. This flux $\Phi$ coexists with $\Phi_{z}$, which is responsible for the emergence of the Weyl points. (b) The energy spectrum of the Weyl semimetal in the presence of $\Phi$. The in-gap gapless modes (denoted by the red lines) connect the gapped Weyl points with opposite chirality. The in-gap gapless mode denotes the energy spectrum formed by the zeroth Landau level.

Fig. 5(b). The in-gap modes represent the energy of the zeroth Landau levels.

With a constant force applied parallel to the magnetic field along the $x$ direction, atoms can be adiabatically pumped from one Weyl point to another Weyl point with opposite chirality. This is the manifestation of chiral anomaly. The chiral anomaly induced atom number imbalance between different Weyl points is expected to cause asymmetric quasimomentum distribution in the time-of-flight measurements [37-39]. Furthermore the recent advancement in cold atom quantum transport measurement [40-42] can enable the study of chiral anomaly induced negative magnetoresistance [43] in atomic systems.

\section{CONCLUSION}

In conclusion, we introduced an experimentally feasible way for realizing Weyl semimetals by applying Raman lasers to coupled multilayer honeycomb optical lattices. We suggest that the measurements of Landau-Zener tunneling probabilities can be used to detect the Weyl semimetal phase, and the possible exploration of chiral anomaly is discussed.

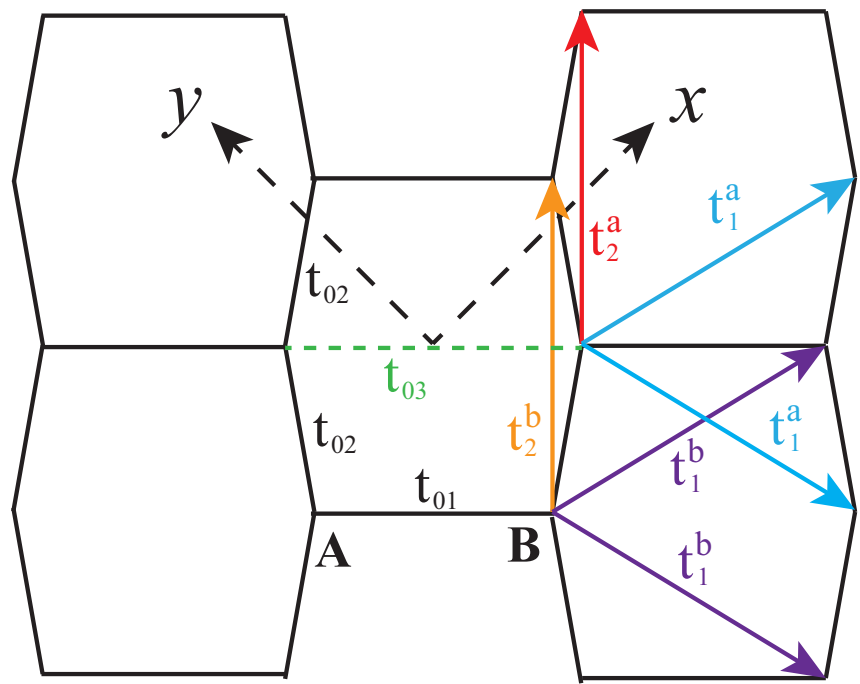

FIG. 6. The hopping terms considered in the honeycomb lattice (brickwall lattice).

Our scheme will lay the foundation for the realization of even more exotic nodal topological phases such as Weyl superconductors $[44,45]$ when attractive interactions between atoms are introduced.

Note added. Recently, we noted that a scheme to realize the Weyl semimetal phase by stacking 2D Harper systems in cubic lattices was proposed [46].

\section{ACKNOWLEDGMENTS}

We acknowledge the support of HKRGC through Grant No. HKUST3/CRF/13G. W.-Y.H. and K.T.L. are further supported by GRF Grants No. 602813, No. 605512, and No. 16303014. W.-Y.H. also acknowledges the support of Hong Kong $\mathrm{PhD}$ Fellowship. S.Z.Z. is further supported by HKRGC Grant No. HKU709313P.

\section{APPENDIX A: TIGHT-BINDING MODEL}

Coupled by the Raman field, the periodic drive system involving the resonant modulation can be described by a time-independent effective Hamiltonian [30,31]. With hopping terms indicated in Fig. 6, the Hamiltonian reads

$$
\begin{aligned}
H= & \sum_{m, n, l} t_{1}^{a}\left(a_{m-1, n, l}^{\dagger} a_{m, n, l}+a_{m, n-1, l}^{\dagger} a_{m, n, l}\right)+t_{2}^{a} a_{m-1, n+1, l}^{\dagger} a_{m, n, l}+t_{\perp} a_{m, n, l-1}^{\dagger} a_{m, n, l}+t_{1}^{b}\left(b_{m-1, n, l}^{\dagger} b_{m, n, l}+b_{m, n-1, l}^{\dagger} b_{m, n, l}\right) \\
& +t_{2}^{b} b_{m-1, n+1, l}^{\dagger} b_{m, n, l}+t_{\perp} b_{m, n, l-1}^{\dagger} b_{m, n, l}+\left[2 V_{0} \sin \left(\Delta \mathbf{p} \cdot \mathbf{r}_{m, n, l}^{a}-\delta \omega t\right)-\frac{\Delta}{2}\right] a_{m, n, l}^{\dagger} a_{m, n, l} \\
& +\left[2 V_{0} \sin \left(\Delta \mathbf{p} \cdot \mathbf{r}_{m, n, l}^{b}-\delta \omega t\right)+\frac{\Delta}{2}\right] b_{m, n, l}^{\dagger} b_{m, n, l}+t_{02}\left(a_{m, n, l}^{\dagger} b_{m-1, n, l}+a_{m, n, l}^{\dagger} b_{m, n-1, l}\right)+t_{01} a_{m, n, l}^{\dagger} b_{m, n, l}++ \text { H.c. },
\end{aligned}
$$

where $r_{m, n, l}^{a}=m \mathbf{v}_{1}+n \mathbf{v}_{2}+l_{\mathbf{d}}$ and $r_{m, n, l}^{b}=m \mathbf{v}_{1}+\mathbf{v}_{2}+l \mathbf{d}+\mathbf{R}_{b}-\mathbf{R}_{a}$. Considering the gauge transformation

$$
U=\sum_{m, n, l} \exp \left(\frac{1}{2} i \delta \omega t\right) a_{m, n, k}^{\dagger} a_{m, n, k}+\exp \left(-\frac{1}{2} i \delta \omega t\right) b_{m, n, k}^{\dagger} b_{m, n, k}
$$


we have

$$
H^{\prime}=U^{\dagger} H U-i \hbar U^{\dagger} \frac{d U}{d t}=H_{0}+H_{1} e^{i \delta \omega t}+H_{-1} e^{-i \delta \omega t}
$$

with

$$
\begin{aligned}
& H_{0}= \sum_{m, n, l} t_{1}^{a}\left(a_{m-1, n, l}^{\dagger} a_{m, n, l}+a_{m, n-1, l}^{\dagger} a_{m, n, l}\right)+t_{2}^{a} a_{m-1, n+1, l}^{\dagger} a_{m, n, l}+t_{\perp} a_{m, n, l-1}^{\dagger} a_{m, n, l}+t_{1}^{b}\left(b_{m-1, n, l}^{\dagger} b_{m, n, l}+b_{m, n-1, l}^{\dagger} b_{m, n, l}\right) \\
&+t_{2}^{b} b_{m-1, n+1, l}^{\dagger} b_{m, n, l}+t_{\perp} b_{m, n, l-1}^{\dagger} b_{m, n, l}+\epsilon\left(a_{m, n, l}^{\dagger} a_{m, n, l}-b_{m, n, l}^{\dagger} b_{m, n, l}\right)+\text { H.c., } \\
& H_{1}=\sum_{m, n, l} t_{01} b_{m, n, l}^{\dagger} a_{m, n, l}+t_{02}\left(b_{m-1, n, k}^{\dagger} a_{m, n, k}+b_{m, n-1, k}^{\dagger} a_{m, n, k}\right)+V_{0} e^{-i\left(\Delta \mathbf{p} \cdot \mathbf{r}_{m, n, l}^{a}-\frac{\pi}{2}\right)} a_{m, n, l}^{\dagger} a_{m, n, l}+V_{0} e^{-i\left(\Delta \mathbf{p} \cdot \mathbf{r}_{m, n, l}^{b}-\frac{\pi}{2}\right)} b_{m, n, l}^{\dagger} b_{m, n, l},
\end{aligned}
$$

and

$$
H_{-1}=\sum_{m, n, l} t_{01} a_{m, n, l}^{\dagger} b_{m, n, l}+t_{02}\left(a_{m-1, n, k}^{\dagger} b_{m, n, k}+a_{m, n-1, k}^{\dagger} b_{m, n, k}\right)+V_{0} e^{i\left(\Delta \mathbf{p} \cdot \mathbf{r}_{m, n, l}^{a} l^{\frac{\pi}{2}}\right)} a_{m, n, l}^{\dagger} a_{m, n, l}+V_{0} e^{i\left(\Delta \mathbf{p} \cdot \mathbf{r}_{m, n, l}^{b} l^{\left.\frac{\pi}{2}\right)}\right.} b_{m, n, l}^{\dagger} b_{m, n, l} .
$$

Here we consider the effective Hamiltonian to the first order:

$$
H_{\mathrm{eff}}=H_{0}+\frac{1}{\hbar \omega}\left[H_{1}, H_{-1}\right]+O\left(\frac{1}{\omega^{2}}\right)
$$

then

$$
\begin{aligned}
H_{\mathrm{eff}}^{(1)}= & \frac{1}{\hbar \omega} \sum_{m, n, l}\left(t_{01}^{2}+2 t_{02}^{2}\right) b_{m, n, l}^{\dagger} b_{m, n, l}+t_{01} t_{02}\left(b_{m, n, l}^{\dagger} b_{m-1, n, l}+b_{m, n, l}^{\dagger} b_{m, n-1, l}\right)+t_{02}^{2} b_{m, n, l}^{\dagger} b_{m+1, n-1, l}-\left(t_{01}^{2}+2 t_{02}^{2}\right) a_{m, n, l}^{\dagger} a_{m, n, l} \\
& -t_{01} t_{02}\left(a_{m, n, l}^{\dagger} a_{m-1, n, l}+a_{m, n, l}^{\dagger} a_{m, n-1, l}\right)-t_{02}^{2} a_{m, n, l}^{\dagger} a_{m+1, n-1, l}+2 t_{02} V_{m-1, n}^{m, n} e^{-i \frac{1}{2} \Delta \mathbf{p} \cdot\left(\mathbf{r}_{m, n, l}^{a}+\mathbf{r}_{m-1, n, l}^{b}\right)} a_{m, n, l}^{\dagger} b_{m-1, n, l} \\
& +2 t_{02} V_{m-1, n}^{m, n} e^{\left.i \frac{1}{2} \Delta \mathbf{p} \cdot \mathbf{r}_{m, n, l}^{a}+\mathbf{r}_{m-1, n, l}^{b}\right)} b_{m-1, n, l}^{\dagger} a_{m, n, l}+2 t_{02} V_{m, n-1}^{m, n} e^{-i \frac{1}{2} \Delta \mathbf{p} \cdot\left(\mathbf{r}_{m, n, l}^{a} l \mathbf{r}_{m, n-1, l}^{b}\right)} a_{m, n, l}^{\dagger} b_{m, n-1, l} \\
& +2 t_{02} V_{m, n-1}^{m, n} e^{i \frac{1}{2} \Delta \mathbf{p} \cdot\left(\mathbf{r}_{m, n, l}^{a}+\mathbf{r}_{m, n-1, l}^{b}\right)} b_{m, n-1, l}^{\dagger} a_{m, n, l}+2 t_{01} V_{m, n}^{m, n} e^{-i \frac{1}{2} \Delta \mathbf{p} \cdot\left(\mathbf{r}_{m, n, l}^{a}+\mathbf{r}_{m, n, l}^{b}\right)} a_{m, n, l}^{\dagger} b_{m, n, l} \\
& +2 t_{01} V_{m, n}^{m, n} e^{i \frac{1}{2} \Delta \mathbf{p} \cdot\left(\mathbf{r}_{m, n, l}^{a}+\mathbf{r}_{m, n, l}^{b}\right)} b_{m, n, l}^{\dagger} a_{m, n, l},
\end{aligned}
$$

where $V_{m^{\prime}, n^{\prime}}^{m, n}=V_{0} \sin \frac{1}{2} \Delta \mathbf{p} \cdot\left(\mathbf{r}_{m, n, l}^{a}-\mathbf{r}_{m^{\prime}, n^{\prime}, l}^{b}\right)$. It is clear that the Raman lasers will introduce the Peierls phase $\exp \left[i \Delta \mathbf{p} \cdot\left(\mathbf{r}+\mathbf{r}^{\prime}\right) / 2\right]$. For the ideal model in the main text, we assume $t_{1}^{a}=t_{1}^{b}=t_{1}, t_{2}^{a}=t_{2}^{b}=t_{2}$ and ignore the difference in $A A$ and $B B$ sublattice hopping in the effective Hamiltonian. For $\Delta \mathbf{p}=\left(0, p_{y}, p_{z}\right),\left|\sin \Delta \mathbf{p} \cdot\left(\mathbf{r}_{i, l}-\mathbf{r}_{j, l}\right)\right|$ has the same value for the nearest-neighbor hopping, so the total Hamiltonian is simplified as

$$
H=\sum_{\langle i, j\rangle, l}\left[t_{i j}^{0} e^{i \frac{1}{2} \Delta \mathbf{p} \cdot\left(\mathbf{r}_{i, l}^{a}+\mathbf{r}_{j, l}^{b}\right)} b_{i, l}^{\dagger} a_{j, l}+t_{i j}^{a} a_{i, l}^{\dagger} a_{j, l}+t_{i j}^{b} b_{i, l}^{\dagger} b_{j, l}\right]+\epsilon \sum_{i, l}\left(a_{i, l}^{\dagger} a_{i, l}-b_{i, l}^{\dagger} b_{i, l}\right)+\sum_{i, l}\left(t_{\perp}^{a} a_{i, l}^{\dagger} a_{i, l+1}+t_{\perp}^{b} b_{i, l}^{\dagger} b_{i, l+1}\right)+\text { H.c. }
$$

The spatial dependence of the Peierls phases is eliminated through the unitary transformation as follows:

$$
\begin{aligned}
& \tilde{a}_{i, l}=a_{i, l} \exp \left(i \frac{1}{2} \Delta \mathbf{p} \cdot \mathbf{r}_{i, l}^{a}\right), \\
& \tilde{b}_{j, l}=b_{j, l} \exp \left(-i \frac{1}{2} \Delta \mathbf{p} \cdot \mathbf{r}_{j, l}^{b}\right) .
\end{aligned}
$$

After the gauge transformation, the new Hamiltonian takes the form

$$
\begin{aligned}
H= & \sum_{\langle i, j\rangle, l}\left(t_{i j}^{0} \tilde{b}_{i, l}^{\dagger} \tilde{a}_{j, l}+t_{i j}^{a} e^{i \phi_{i j}} \tilde{a}_{i, l}^{\dagger} \tilde{a}_{j, l}+t_{i j}^{b} e^{-i \phi_{i j}} \tilde{b}_{i, l}^{\dagger} \tilde{b}_{j, l}\right) \\
& +\epsilon \sum_{i, l}\left(\tilde{a}_{i, l}^{\dagger} \tilde{a}_{i, l}-\tilde{b}_{i, l}^{\dagger} \tilde{b}_{i, l}\right)+\sum_{i, l}\left(t_{\perp}^{a} e^{i \phi_{\perp}} \tilde{a}_{i, l}^{\dagger} \tilde{a}_{i, l+1}\right. \\
& \left.+t_{\perp}^{b} e^{-i \phi_{\perp}} \tilde{b}_{i, l}^{\dagger} \tilde{b}_{i, l+1}\right)+ \text { H.c. }
\end{aligned}
$$

Further labeling $A(B)$ sublattices as corresponding to a pseudospin $\sigma$, the Hamiltonian is transformed in $\mathbf{k}$ space as follows:

$$
H(\mathbf{k})=\left(\begin{array}{ll}
h_{11} & h_{12} \\
h_{21} & h_{22}
\end{array}\right)=d_{0}(\mathbf{k}) I+\mathbf{d}(\mathbf{k}) \cdot \sigma .
$$

Weyl points will emerge when the conditions $d_{x}(\mathbf{k})=d_{y}(\mathbf{k})=$ $d_{z}(\mathbf{k})=0$ are satisfied. For the case $\left|t_{i j}^{0}\right|=t, d_{x}(\mathbf{k})=d_{y}(\mathbf{k})=$ 0 occurs when $\left(k_{x} a, k_{y} a\right)=\left(-\frac{2}{3} \pi,-\frac{5}{3} \pi\right)$ or $\left(k_{x} a, k_{y} a\right)=$ $\left(\frac{2}{3} \pi,-\frac{1}{3} \pi\right)$. Thus the critical condition for the emergence of Weyl points can be determined by judging the existence of real roots for $d_{z}\left(-\frac{5}{3} \pi,-\frac{2}{3} \pi, k_{z}\right)=0$ or $d_{z}\left(-\frac{1}{3} \pi, \frac{2}{3} \pi, k_{z}\right)=0$ with given $\epsilon$ and $\phi_{\perp}$.Physically the existence of Weyl points 

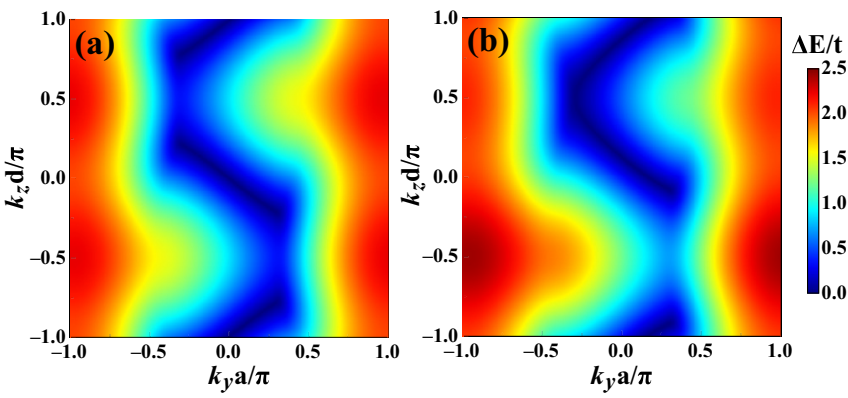

FIG. 7. The Fermi arc on the (100) surfaces of Weyl semimetals. (a) Using the parameters corresponding to Fig. 2(c). (b) Using the parameters corresponding to Fig. 2(d) in the main text.

corresponds to a transition from the topological nontrivial state to the topological trivial state when atoms move along the $k_{z}$ direction. The Fermi arc evolution is shown in Fig. 7.

\section{APPENDIX B: WEYL POINTS IN THE REALISTIC OPTICAL POTENTIAL}

The realistic optical lattice potential with $V_{[\bar{X}, X, Y]}=$ $[7,0.5,2] E_{R}$ and $\theta=\frac{2}{3} \pi$ is considered in order to calculate the band structure for an isolated layer and its corresponding maximally localized Wannier function [47]. The Wannier function $W(x, y, z)=w(x, y) w(z)$ is controlled by the in-plane and out-of-plane optical potential, respectively, and $w(x, y)$ is shown in Fig. 8 with intralayer hopping parameters $\left[t_{01}, t_{02}, t_{03}, t_{1}^{a}, t_{2}^{a}, t_{1}^{b}, t_{2}^{b}\right]=$

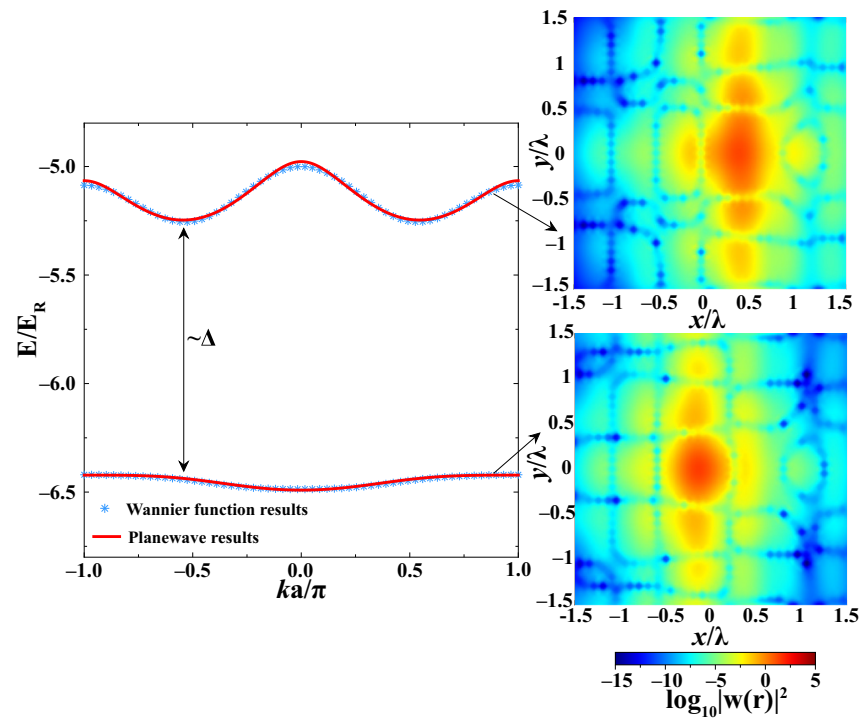

FIG. 8. The band structure and its corresponding maximally localized Wannier function for the lattice potential with $V_{\bar{X}, X, Y}=$ $[7,0.5,2] E_{R}$ and $\theta=\frac{2}{3} \pi$. The hopping terms obtained from the maximally localized Wannier function make the energy spectrum agree well with that from plane-wave results. $\mathbf{k}$ is along $k_{x}=k_{y}$. $\lambda=\frac{2 \pi}{q}=\sqrt{2} a$ is the wavelength of the in-plane laser.

$10^{-2}[-8.29,-1.65,0,0.45,1.86,-0.21,3.22] E_{R}$ and the onsite potential $\Delta=-1.2 E_{R}$. In the resonant case $\Delta=\delta \omega$, we can select the wavelength for the $z$ direction standing wave to have $p_{z} d=\pi$ and make $V_{0}=E_{R}$. Such an optical potential can generate Weyl points as is shown in Fig. 2(b).
[1] M. Z. Hasan and C. L. Kane, Rev. Mod. Phys. 82, 3045 (2010).

[2] X. L. Qi and S. C. Zhang, Rev. Mod. Phys. 83, 1057 (2011).

[3] C.-Z. Chang, J. Zhang, X. Feng, J. Shen, Z. Zhang, M. Guo, K. Li, Y. Ou, P. Wei, L.-L. Wang et al., Science 340, 167 (2013).

[4] X. Wan, A. M. Turner, A. Vishwanath, and S. Y. Savrasov, Phys. Rev. B 83, 205101 (2011).

[5] A. A. Burkov and L. Balents, Phys. Rev. Lett. 107, 127205 (2011).

[6] J. H. Jiang, Phys. Rev. A 85, 033640 (2012).

[7] L. Lu, L. Fu, J. D. Joannopoulos, and M. Soljačić, Nat. Photonics 7, 294 (2013).

[8] Y. Xu, R.-L. Chu, and C. Zhang, Phys. Rev. Lett. 112, 136402 (2014).

[9] A. A. Zyuzin and A. A. Burkov, Phys. Rev. B 86, 115133 (2012).

[10] Z. Wang and S.-C. Zhang, Phys. Rev. B 87, 161107 (2013).

[11] A. C. Potter, I. Kimchi, and A. Vishwanath, Nat. Commun. 5, 5161 (2014).

[12] S.-Y. Xu, C. Liu, S. K. Kushwaha, R. Sankar, J. W. Krizan, I. Belopolski, M. Neupane, G. Bian, N. Alidoust, T.-R. Chang et al., Science 347, 294 (2015).

[13] B. Q. Lv, H. M. Weng, B. B. Fu, X. P. Wang, H. Miao, J. Ma, P. Richard, X. C. Huang, L. X. Zhao, G. F. Chen et al., Phys. Rev. X 5, 031013 (2015).
[14] E. Alba, X. Fernandez-Gonzalvo, J. Mur-Petit, J. K. Pachos, and J. J. García-Ripoll, Phys. Rev. Lett. 107, 235301 (2011); N. Goldman, E. Anisimovas, F. Gerbier, P. Öhberg, I. B. Spielman, and G. Juzeliūnas, New J. Phys. 15, 013025 (2013); E. Anisimovas, F. Gerbier, T. Andrijauskas, and N. Goldman, Phys. Rev. A 89, 013632 (2014).

[15] N. Goldman, I. Satija, P. Nikolic, A. Bermudez, M. A. MartinDelgado, M. Lewenstein, and I. B. Spielman, Phys. Rev. Lett. 105, 255302 (2010).

[16] B. Béri and N. R. Cooper, Phys. Rev. Lett. 107, 145301 (2011).

[17] X.-J. Liu, K. T. Law, and T. K. Ng, Phys. Rev. Lett. 112, 086401 (2014).

[18] X.-J. Liu, X. Liu, C. Wu, and J. Sinova, Phys. Rev. A 81, 033622 (2010)

[19] M. Aidelsburger, M. Atala, M. Lohse, J. T. Barreiro, B. Paredes, and I. Bloch, Phys. Rev. Lett. 111, 185301 (2013); H. Miyake, G. A. Siviloglou, C. J. Kennedy, W. C. Burton, and W. Ketterle, ibid. 111, 185302 (2013).

[20] C. J. Kennedy, G. A. Siviloglou, H. Miyake, W. C. Burton, and W. Ketterle, Phys. Rev. Lett. 111, 225301 (2013).

[21] P. Hauke, O. Tieleman, A. Celi, C. Ölschläger, J. Simonet, J. Struck, M. Weinberg, P. Windpassinger, K. Sengstock, M. Lewenstein, and A. Eckardt, Phys. Rev. Lett. 109, 145301 (2012). 
[22] W. Zheng and H. Zhai, Phys. Rev. A 89, 061603(R) (2014).

[23] M. D. Reichl and E. J. Mueller, Phys. Rev. A 89, 063628 (2014).

[24] G. Jotzu, M. Messer, R. Desbuquois, M. Lebrat, T. Uehlinger, D. Greif, and T. Esslinger, Nature (London) 515, 237 (2014).

[25] M. Aidelsburger, M. Lohse, C. Schweizer, M. Atala, J. T. Barreiro, S. Nascimbène, N. R. Cooper, I. Bloch, and N. Goldman, Nat. Phys. 11, 162 (2015).

[26] A. Bermudez, L. Mazza, M. Rizzi, N. Goldman, M. Lewenstein, and M. A. Martin-Delgado, Phys. Rev. Lett. 105, 190404 (2010).

[27] S.-T. Wang, D.-L. Deng, and L.-M. Duan, Phys. Rev. Lett. 113, 033002 (2014); D.-L. Deng, S.-T. Wang, and L.-M. Duan, Phys. Rev. A 90, 041601(R) (2014).

[28] L. Tarruell, D. Greif, T. Uehlinger, G. Jotzu, and T. Esslinger, Nature (London) 483, 302 (2012).

[29] T. Uehlinger, G. Jotzu, M. Messer, D. Greif, W. Hofstetter, U. Bissbort, and T. Esslinger, Phys. Rev. Lett. 111, 185307 (2013).

[30] D. Jaksch and P. Zoller, New J. Phys. 5, 56 (2003).

[31] N. Goldman, J. Dalibard, M. Aidelsburger, and N. R. Cooper, Phys. Rev. A 91, 033632 (2015).

[32] H. M. Price and N. R. Cooper, Phys. Rev. A 85, 033620 (2012).

[33] X.-J. Liu, K. T. Law, T. K. Ng, and P. A. Lee, Phys. Rev. Lett. 111, 120402 (2013).

[34] D. Xiao, M.-C. Chang, and Q. Niu, Rev. Mod. Phys. 82, 1959 (2010).

[35] L.-K. Lim, J.-N. Fuchs, and G. Montambaux, Phys. Rev. Lett. 108, 175303 (2012).
[36] H. B. Nielsen and M. Ninomiya, Phys. Lett. B 130, 389 (1983).

[37] J. Struck, C. Olschlager, M. Weinberg, P. Hauke, J. Simonet, A. Eckardt, M. Lewenstein, K. Sengstock, and P. Windpassinger, Phys. Rev. Lett. 108, 225304 (2012)

[38] B. K. Stuhl, H.-I. Lu, L. M. Aycock, D. Genkina, and I. B. Spielman, Science 349, 1514 (2015).

[39] M. Mancini, G. Pagano, G. Cappellini, L. Livi, M. Rider, J. Catani, C. Sias, P. Zoller, M. Inguscio, M. Dalmonte, and L. Fallani, Science 349, 1510 (2015).

[40] C.-C. Chien, S. Peotta and M. Di Ventra, Nat. Phys. 11, 998 (2015).

[41] J.-P. Brantut, J. Meineke, D. Stadler, S. Krinner, and T. Esslinger, Science 337, 1069 (2012).

[42] S. Krinner, D. Stadler, D. Husmann, J.-P. Brantut, and T. Esslinger, Nature (London) 517, 64 (2015).

[43] X. Huang, L. Zhao, Y. Long, P. Wang, D. Chen, Z. Yang, H. Liang, M. Xue, H. Weng, Z. Fang, X. Dai, and G. Chen, Phys. Rev. X 5, 031023 (2015).

[44] Tobias Meng and Leon Balents, Phys. Rev. B 86, 054504 (2012).

[45] B. Liu, X. Li, L. Yin, and W. V. Liu, Phys. Rev. Lett. 114, 045302 (2015).

[46] T. Dubcek, C. J. Kennedy, L. Lu, W. Ketterle, M. Soljacic, and H. Buljan, Phys. Rev. Lett. 114, 225301 (2015).

[47] R. Walters, G. Cotugno, T. H. Johnson, S. R. Clark, and D. Jaksch, Phys. Rev. A 87, 043613 (2013). 